\title{
The impact of malnutrition on health care use in hospital outpatients
}

\author{
A. L. Cawood ${ }^{1}$, S. Rust ${ }^{2}$, E. Walters ${ }^{2}$, R. J. Stratton ${ }^{1}$ and M. Elia ${ }^{3}$ \\ ${ }^{1}$ Medical Affairs, Nutricia Ltd, Trowbridge, BA14 OXQ, UK, ${ }^{2}$ Nutrition and Dietetic Department, Southampton General \\ Hospital, Southampton SO16 6YD, UK and ${ }^{3}$ Institute of Human Nutrition, University of Southampton, Tremona Road, \\ Southampton SO16 6YD, UK
}

Malnutrition is common and costly, yet remains under-recognised and under-treated ${ }^{(1)}$. Previous studies have shown that the 'Malnutrition Universal Screening Tool' ('MUST') $)^{(2)}$ predicts clinical outcome in hospitalised elderly ${ }^{(3)}$; however, the clinical consequences of malnutrition in outpatients has not been investigated.

One hundred and ninety four outpatients (age 54 (SD 17.19) years; $45 \%$ female) were screened for malnutrition using 'MUST' at Southampton General Hospital between July 2008 and June 2009. Healthcare use was collected prospectively during the subsequent 6 months (hospital admissions (planned and emergency), length of hospital stay and outpatient appointments). The patients attended a range of outpatient clinics (40\% gastroenterology, $21 \%$ surgical, $17 \%$ medical, $8 \%$ oncology and $14 \%$ other). Eighteen percent were at risk of malnutrition ( $12 \%$ medium and $6 \%$ high risk). Age and sex were not significantly related to malnutrition risk.

Outpatients at risk of malnutrition experienced significantly more hospital admissions (planned and emergency) and had a significantly longer length of hospital stay (table). The use of healthcare resources progressively increased from low to medium and high risk of malnutrition (table).

\begin{tabular}{lcrr}
\hline & \multicolumn{3}{c}{ 'MUST' } \\
\cline { 2 - 4 } & Low risk $(n=159)$ & Med risk $(n=23)$ & High risk $(n=12)$ \\
\hline All hospital admissions length of stay (days) & $0.90 \pm 3.9$ & $2.04 \pm 4.9$ & $4.92 \pm 8.1$ \\
\% Hospital admissions in 6 months & $12.6 \%$ & $26.1 \%$ & $66.7 \%$ \\
\% Emergency admissions in 6 months & $5.0 \%$ & $8.7 \%$ & $41.7 \%$ \\
\% Planned admissions in 6 months & $7.5 \%$ & $21.7 \%$ & $0.007 \#$ \\
\% Outpatient appointments in 6 months & $65.4 \%$ & $82.6 \%$ & $0.000^{*}$ \\
\hline
\end{tabular}

Med = medium risk; \# = statistically significant ANOVA. Length of stay mean \pm SD

$*$ Statistically significant; $\mathrm{Chi}^{2}$ test; † statistically significant $(P($ trend $)=0.018)$.

The impact of malnutrition on admissions remained significant even when adjusted for age. Six month mortality was higher in at risk patients $(5.7 \%)$ compared to low risk $(2.5 \%)$ although this was not significant $\left(P=0.322, \mathrm{Chi}^{2}\right)$.

This study shows that malnutrition in general outpatients is associated with increased health care use. Identifying and appropriately treating malnutrition could potentially reduce health care costs.

1. Elia M \& Russell C (2009) Combating Malnutrition: Recommendations for Action. Redditch, Worcs.: BAPEN.

2. Elia M (2003) The 'MUST' Report. Redditch, Worcs.: BAPEN (http://www.bapen.org.uk).

3. Stratton RJ et al. (2006) Br J Nutr 95, 325-330. 